\title{
A Matter of Rules? A Longitudinal Study of Parents' Influence on Young People's Drinking Trajectories
}

\author{
Jeanette Østergaard ${ }^{a}$ Margaretha Järvinen ${ }^{b} \quad$ Asger Graa Andreasen $^{a}$ \\ ${ }^{a}$ VIVE - The Danish Center for Social Science Research, Copenhagen, Denmark; ${ }^{b}$ Department of Sociology, \\ University of Copenhagen, Copenhagen, Denmark
}

\section{Keywords}

Adolescents and alcohol · Parental rules · Heavy episodic drinking · Longitudinal surveys

\begin{abstract}
Based on longitudinal survey data $(2005,2008,2015)$, this paper investigates binge drinking among young people in Denmark. We analyse the relationship between parental alcohol rules in 2005 and the development of their children's heavy episodic drinking from age 15 to 25/26 using a multilevel approach to repeated measures. Two hypotheses are tested. The first is that young people from families with "strict" alcohol rules have a lower level of binge drinking than young people from families with lenient rules. However, given the cultural pressure on adolescents to drink heavily in Denmark, we also expect parents to face challenges when trying to limit their children's drinking. Hence, our second hypothesis is that youths with strict alcohol rules at age 15 show the steepest increase in heavy episodic drinking when going from early to late adolescence and thus gradu-
\end{abstract}

(c) 2018 S. Karger AG, Basel ally catch up with young people who had lenient rules. Both our hypotheses are confirmed: Strict alcohol-specific rules are associated with lower rates of binge drinking, but with time young people with strict rules close in on their peers' alcohol use.

(c) 2018 S. Karger AG, Basel

\section{Introduction}

There is a long tradition of research addressing the question how parents can influence their adolescents' relationship to alcohol. In previous decades, this research was dominated by US studies examining if parental rules, typically prohibition of alcohol consumption before the age of 18, could postpone adolescents' alcohol debut and/ or curb the intensity of their drinking - with several studies showing an association between rules and drinking patterns [1-5]. While most of this research was cross-sectional, a few studies were longitudinal. For instance, Guo et al. [6] followed a sample of 808 youths in Seattle from

\section{KARGER}

E-Mail karger@karger.com www.karger.com/ear
Jeanette Østergaard

VIVE - The Danish Center for Social Science Research

Herluf Trolles Gade 11

DK-1052 Copenhagen K (Denmark)

E-Mail jea@vive.dk 
age 10 to 21 . They identified a variety of factors at age 10 , 14 , and 16 that were protective in relation to heavy alcohol use at age 21 - one of them being parental monitoring and family rules at age 16 . Other protective factors were bonding to school, high educational expectations and low alcohol use among friends [6].

In the past ten years, a growing number of European studies have been published, addressing the question whether parental alcohol rules have a protective effect also in countries where adolescent drinking is legal and intoxication is an integrated part of mainstream youth cultures. In a Dutch study, Van der Vorst et al. [7] gathered data on drinking rules and alcohol consumption from a sample of parents and their adolescents (aged 1316). The study showed that parental alcohol rules (essentially non-acceptance of teenage drinking) lowered the likelihood of adolescents' drinking initiation but that the impact of rules declined once the adolescents had established a drinking pattern with their peers. In another Dutch study, Janssen et al. [8] confirmed that alcohol rules delay drinking onset, and also that strict alcoholspecific parenting decreases the odds of drinking heavily among 13-16-year-olds (see also $[9,10]$ ). While the studies of van der Vorst et al. [7, 9] and Janssen et al. [8] followed adolescents' drinking over a couple of years only, Mares et al. [11] report covers a longer time-span. A sample of adolescents from 428 Dutch families was followed from when they were age 13 to 20 . The study showed first that adolescent drinking increased gradually from early to late adolescence, and second that the strictness of alcohol-specific rules at a certain point of time predicted the intensity of drinking one year later throughout the period [11].

In the United Kingdom, researchers have also looked at the impact of parental rule setting. In a study of 12-16-year-olds in Northern Ireland, McKay [12] found that adolescents' perceptions of parental alcohol rules were significantly associated with their self-reported alcohol use, even when variables such as attachment to parents, attachment to peers, school grade and gender were controlled for. Similarly, Miller and Plant's [13] British study showed that young people describing their parents as favourable towards alcohol use reported more experiences with drunkenness than young people saying that their parents discouraged alcohol use. In general, parental guidance was associated with less drinking also when background variables (e.g., parents' education and closeness of parent-child relationship) were controlled for. Like other research areas in the field, this British study was based on adolescents' descriptions of their parents'

A Longitudinal Study of Parents' Influence on Young People's Drinking Trajectories alcohol rules and attitudes, and not on survey answers from the parents themselves.

The present study investigates the effects of parental alcohol rule setting in Denmark, a country with a drunkenness-focused alcohol culture. For several reasons, Denmark is an informative case to study the longterm effect of alcohol rules, here defined as rules parents impose on their children when they are partying with friends (see [11] for a similar definition).

First, comparative research consistently shows that Danish adolescents are at the international top of countries when it comes to intoxication. For instance, $34 \%$ of Danish 15-year-olds in 2003 (about the same cohort that we focus on here) reported they had been drunk 10 times or more during the past 12 months, the corresponding figures in some other countries being $7 \%$ in the United States, $11 \%$ in Germany, $15 \%$ in Sweden and 25\% in the United Kingdom [14]. Comparative research on occasional heavy drinking among adults shows a similar international pattern. A Eurobarometer study of alcohol use in 27 European countries lists Denmark at the top (together with Ireland and Finland) when it comes to binge drinking in the general population (15+). Answering the question "On a day when you drink, how much do you usually drink?," 23\% of Danes answered that they usually consume 5+ units of alcohol. The corresponding figures in low-drunkenness countries such as Italy, Belgium, Spain and Germany varied between 0 and $8 \%$ [15]. These numbers indicate that Danish parents are up against unusually strong cultural forces when they try to limit their children's binge drinking.

Second, young people's drinking in Denmark almost exclusively takes place in social settings (such as private house parties) where no parents are present, and drinking to intoxication is more or less normative behaviour [16]. Thus, it is relevant to investigate whether parents' strict rules can be transferred to and have an effect in peersonly drinking sessions where drunkenness-focused alcohol consumption is seen as a natural part of socialisation.

We use longitudinal data from 2005, 2008 and 2015 to investigate whether Danish parents' attempts at restricting their children's alcohol intake in 2005 are related to a lower level of binge drinking in 2005-2015. We combine survey data with high-quality administrative data about family income and parents' education in order to get as precise information as possible about the young people's family background. Contrary to much previous research in this field, we use parents' descriptions of alcohol rules (and not their children's reports on these rules). Hence, we are able to separate parental sur- 
vey answers about rules from adolescents' self-reported drinking practices. We define binge drinking (used synonymously with "heavy episodic drinking") as consumption of 5 or more units of alcohol on one drinking occasion.

Young people's drinking levels may of course be influenced by a wide range of other factors (family-related and others) than alcohol rules. Previous studies have pointed to variables such as parents' education and income, parental divorce, parental attitudes to alcohol, parents' own drinking, the young people's education, and their civil and family status (for reviews, see [17-20]). When investigating the relationship between parental rules and young people's drinking patterns, we will control for these factors.

Two hypotheses are tested. The first is that young people from families with "strict" alcohol rules in 2005 have a lower level of binge drinking in the period 2005-2015 than young people from families with more lenient rules. The second hypothesis is that youths with strict rules show the steepest increase in heavy episodic drinking from age 15 to age $18 / 19$, and thus at age $25 / 26$ reach the level of their peers.

The first hypothesis is inspired by international research on parental rule-setting; we want to investigate if alcohol-specific rules have an effect also in Denmark. The second hypothesis is motivated by Denmark's leading position as a drunkenness-focused country. We expect cultural forces to work against the attempts of some parents to regulate their adolescents' drinking. Our own previous research has shown that a majority of Danish parents and their children - define adolescent binge drinking as a "natural" part of growing up [21]. We therefore expect that cautious young drinkers in our sample are under considerable pressure to adapt their drinking to that of their peers, especially during late adolescence when drunkenness-focused drinking peaks.

\section{Data and Methods}

This study builds on a 3-wave longitudinal survey of young people's alcohol and drug use. The young people were interviewed at age 15 (2005), 18/19 (2008), 25/26 (2015), and at baseline a cross-sectional survey with the youths' parents was also conducted. To sample the young people and their parents at baseline, we used the unique Danish Civil Registration System whereby 2,000 children born in 1989 were randomly selected from all over the country.

The surveys were linked through the respondents' unique civil registration numbers to extensive national administrative data on the Danish population. At baseline (2005), the surveys with the young people and their parents were conducted as postal surveys. In 2008 and 2015, the survey with young people was conducted both as a postal and Internet survey.

The study was approved by the Danish Data Protection Agency and follows this agency's standards for contacting survey respondents, storing data and linking survey data to register data. The project also follows the ethical standards of the Danish Research Council for Independent Research. Hence, respondents were informed that participation in the study was voluntary and that all data are being kept highly confidential. All information that could potentially be traced back to individuals was anonymised. Access to the linked survey and register data requires approval from Statistics Denmark, and data was stored on Statistics Denmark's server and handled according to their strict ethical and technical guidelines.

The response rate for young people was $72 \%(n=1,445)$ in 2005. Excluding people who could not be reached due to unknown addresses or because they had left the country, the response rate for the unbalanced data was $63 \%(n=1,144)$ in 2008 and $57 \%$ in $2015(n=1,056)$. The response rate in the 2005 parental survey was $72 \%$. The combined response rate (young people and their parents) in 2005 was $65 \%(n=1,292)$. On this condition, 873 young people answered the survey in 2008 and 784 answered the survey in 2015. Compared to baseline, young people who participated in the follow-up studies in either 2008 or 2015 were more likely to be females $\left(\mathrm{chi}^{2}=13.4, p=0.000\right)$ and to come from families where the father had a relatively high education (measured with the help of administrative data from $2005 ; \mathrm{chi}^{2}=18, p=0.001$ ). No statistical differences were found between follow-up participants and others in terms of binge drinking $\left(\mathrm{chi}^{2}=7.9, p=0.16\right)$, drinking to intoxication $\left(\mathrm{chi}^{2}=0.3, p=0.6\right)$ or abstaining from alcohol $\left(\mathrm{chi}^{2}=\right.$ $0.0, p=0.86)$. Neither was attrition related to differences in parental alcohol rules $\left(\mathrm{chi}^{2}=9.3, p=0.054\right)$.

\section{Measures}

Parental alcohol rules 2005: In the 2005 survey with parents, respondents were asked: How many units of alcohol is your child allowed to drink if he/she is at a party with friends? Answer categories: 0 units, 1 unit, 2 units, 3-4 units, 5+ units, have no rules, don't know. For the analyses, we collapsed 0-2 units into one category - the reference category - as our data showed an important dividing line for young people's heavy episodic drinking at age 15 if their parents allowed them to drink 3 units or more [21]. We are aware that our definition of max 2 units of alcohol as "strict" rules for 15-year-olds may seem odd in an international perspective, especially in countries where adolescent alcohol consumption is illegal. The term "strict" is relative and dependent on the cultural context. In an alcohol culture like Denmark where a majority of adolescents are regular binge drinkers [22, 23], a drinking rule of $\max 2$ units is indeed conceived of as strict by most parents [21]. We will later return to the question of a potential difference between 2 allowed units and 0 units. The answers "have no rules" and "don't know" were included separately. Based on previous analyses of the 2005 study, we interpret them as capturing different aspects of parental rule setting, the former being associated with lenient rules and the latter being most frequent among parents whose children had not yet started to drink.

Parental attitudes 2005: We included 5 statements about upbringing and attitudes to young people's alcohol consumption in 
the 2005 survey with parents. The statements were (1) Parents should be an authority for their children; (2) As long as adolescents live at home, parents may prohibit them from getting drunk; (3) Adolescents who have received a good upbringing are able to set their own limits for their behaviour; (4) It is natural for adolescents to learn to drink in the company of their peers; and (5) The only way adolescents learn the limits for their own alcohol intake is by transgressing them. For all 5 questions, the answer categories were a Likert scale of strongly agree, agree, disagree and strongly disagree. For the statistical analysis, the answers were recoded into binary variables (dummy variables) as an explorative factor analysis revealed that these 5 items on upbringing were not captured by one latent variable [21].

Parents' drinking and smoking in 2005: To measure parental drinking we used the question: How many units of alcohol did you consume the previous week? This was measured by counting total units of consumption for each day of the week. In relation to cigarette smoking, parents were asked whether they were current smokers, ex-smokers or non-smokers. We included this as a dummy variable measuring "never smoked" vs. "smokes" or "has smoked".

Parents' education 2005: We obtained information about parents' education from administrative registers. To avoid multicollinearity, we included only fathers' highest completed education in the model, using 4 educational categories: (1) secondary or upper secondary school only; (2) vocational training; (3) short- or medium-cycle higher education; and (4) long cycle higher education. We use category 1 as the reference category.

Equivalence-scaled family income 2005: We used administrative register data to calculate family income, defined as the family's equivalence-scaled disposable income in 2005. We calculated the equivalence factor in accordance with the Danish Ministry for Economic Affairs and the Interior [24]. The equivalence-scaled income takes into account that it is economically advantageous to live in a household with more people because of the possibility of sharing expenses. In the analyses, we include income as a natural log-transformed continuous variable.

Divorced parents 2005: Information about parental divorce was obtained from the survey with 15 -year-olds, where about $25 \%$ reported that their parents were divorced in 2005.

Young adults' occupational status 2015: We included survey information about the respondents' occupational status at age 25/26 (employed, unemployed, student or other), as previous research suggests that drinking patterns may be related to being a student or having steady employment [25-28].

Young adults in a relationship 2015: We control for whether the respondents were in a relationship in 2005, 2008 or 2015, as previous research also suggests that steady, romantic relationships may influence people's drinking habits [29-31]. Thus, the young adults were asked if they currently had a girlfriend/boyfriend or (in later waves) a spouse. As the relationship status on each measurement occasion may differ, we included the variable as a time-varying predictor.

Binge drinking (heavy episodic drinking) 2005, 2008 and 2015: This outcome variable was measured with an identical question in all 3 surveys with young people: How many times during the past 30 days have you drunk 5 units of alcohol or more on one occasion? The answer categories were 0 times, 1 time, 2 times, 3 times, 4-5 times, 6+ times.

A Longitudinal Study of Parents' Influence on Young People's Drinking Trajectories
Table 1. Percentages of heavy episodic drinking across time

\begin{tabular}{lccc}
\hline & $\begin{array}{c}\text { Age 15/16 } \\
(\text { time }=0)\end{array}$ & $\begin{array}{c}\text { Age 18/19 } \\
\text { (time }=1)\end{array}$ & $\begin{array}{l}\text { Age 25/26 } \\
\text { (time }=2)\end{array}$ \\
\hline 0 times & 55.1 & 21.4 & 29.2 \\
1 time & 18.8 & 20.2 & 28.1 \\
2 times & 10.9 & 19.3 & 19.2 \\
3 times & 6.6 & 16.4 & 11.4 \\
$4+$ times & 8.6 & 22.7 & 12.1 \\
Number & 1,100 & 798 & 686 \\
\hline
\end{tabular}

\section{Statistical Analyses}

To test if heavy episodic drinking among young people develops differently depending on parental rules, we conducted a multilevel regression analysis, (in Stata 14.2) that fits multilevel probit models for ordered responses. A multilevel approach to repeated measures can estimate the trajectories of heavy episodic drinking from 2005 to 2015 . The outcome variable can be thought of as ordinal with increasing categories of heavy episodic drinking. Since young people vary considerably in terms of heavy episodic drinking at both baseline (2005) and across time (Table 1), we use a cumulative ordered probit model with random intercepts and random slopes [32]. To take into account that drinking trajectories are not a linear function of time, we added time squared to the model [33]. Furthermore, we clustered the standard errors of our model on individual identification. Doing so allowed us to take the individual effect of repeated measurement into account without making further parametric assumptions.

As we analyse drinking trajectories using longitudinal data, we allow each individual an intercept (i.e., random intercepts) and growth trajectory (i.e., random slopes), the latter estimating the difference in the rate of heavy episodic drinking among the young people from 2005 to 2015. A random-intercepts model would be too simplistic as it is unlikely that the rate of growth in heavy episodic drinking across time is the same for all individuals. Furthermore, as we will show below, young people's drinking is more similar at the 2 later time points than at baseline, meaning that their alcohol consumption converges over time. To estimate the overlap of individual-specific regression of heavy episodic drinking over time, we included a correlation between the random intercepts and the random slopes. To test whether the frequency of heavy episodic drinking develops differently over time depending on parents' alcohol rules at baseline, we include cross-level interactions between alcohol rules and time (and time squared). Thus, we allow the slopes of the change trajectories in heavy episodic drinking to differ according to parents' rules regarding alcohol consumption.

\section{Results}

\section{Descriptive Statistics}

Table 1 shows the response proportions for our outcome variable, heavy episodic drinking, during the last 10 years with 3 points of data collection, and Table 2 shows the descriptive statistics for the control variables 
that are significant at the 5\% level and are included in the final model. Excluding young people with missing values in one of the variables used in the final statistical model leaves us with 1,100 participants in 2005, 798 in 2008, and 686 in 2015 in our analytical sample.

For simplicity and to avoid sparse cells in the further analyses, we have collapsed heavy episodic drinking of 4-5 times and 6+ times. We can see that across a 10-year time span young people first increase and then lower their frequency of heavy episodic drinking. Thus, at age 15 less than half of the respondents have consumed 5 units or more on at least one drinking occasion within the previous 30 days; at age 18/19 this proportion is almost $80 \%$ and at age $25 / 26$ it is somewhat lower again (around $70 \%$ ). At the same time, the proportion reporting binge drinking 4 times or more during the last 30 days increases from $9 \%$ at age 15 to $23 \%$ at age $18 / 19$ after which it decreases to $12 \%$ at age $25 / 26$. In essence, Table 1 shows that heavy episodic drinking is something that a majority of both 18/19-year-olds and 25/26-year-olds engage in but that frequent binge drinking (3-4 times or more per month) diminishes from late adolescence to young adulthood (from 40 to $24 \%$ ).

In Table 2 we see that in 2005, when the young people were 15-year-olds, a third of the parents had "strict" alcohol rules (maximum 2 units of alcohol). About $10 \%$ of the parents had "lenient" rules (5+ units), while a substantial proportion (25\%) reported they had no rules at all for their children's alcohol consumption. Thus, most parents seem to accept that their children at age 15 consume alcohol when partying with their friends even though the legal age for buying alcohol in Denmark is 16. However, only a minority of parents have very lenient rules.

\section{Multilevel Analyses}

The 2 hypotheses formulated for this paper were first, that youths from families with strict alcohol rules have lower levels of frequent binge drinking than youths from families with lenient rules, and second, that the former group catches up with the latter, especially from age 15 to age $18 / 19$.

The random-coefficient ordered probit model analysis confirmed both hypotheses. As shown in Table 3, lenient rules are associated with higher rates of heavy episodic drinking across time also when we control for socioeconomic background, parents' drinking and attitudes to upbringing (cf. the first hypothesis). However, we also find that binge drinking is associated with several other factors included in the model, the most obvious one being that males report higher levels of heavy episodic drinking
Table 2. Description of independent variables included in the final model

Percent

Parental survey 2005

Parents' alcohol-specific rules 2005

$0-2$ units of alcohol

32.6

3-4 units of alcohol

29.4

$5+$ units

9.5

No rules

25.0

Don't know

3.6

Parents' attitudes 2005

Agree that teenagers with a proper upbringing are able to set limits for themselves

Parents' alcohol consumption 2005

Average number of units $($ min value $=0$; $\max$ value $=30)$

Standard deviation

Survey with young adults

Males

50.2

Register data 2005

Family income 2005

Average household disposable income, natural log $\quad 12.12$

Standard deviation

0.42

Number

1,100

compared to females. Of the 5 statements intended to measure parental attitudes to upbringing in 2005, only one showed a significant association with adolescents' binge drinking. Young people whose parents stressed the significance of "good upbringing" for the development of self-control have lower frequencies of binge drinking than other youths. We also find that household disposable income has a significant positive impact on how adolescents' heavy episodic drinking develops. Thus, if young people come from families with a high household income in contrast to a low, they are more likely to report frequent heavy episodic drinking. Parents' own drinking (as reported in the 2005 parental survey) is also positively associated with their children's binge drinking. On the other hand, parents' education, parental divorce in 2005, and the young adults' occupational situation (employment, unemployment, studies) in 2015 are not statistically significant in the model and thus not related to the development of binge drinking.

As the random intercept is statistically significant, it reveals that young people drink differently (e.g., have different frequencies of heavy drinking) at baseline and as the random slope is also significant, it means that across time, young people's individual growth curves of heavy drinking are different. Furthermore, as the correlation 
Table 3. Mixed effects ordered probit model

\begin{tabular}{|c|c|c|}
\hline Random slope model assuming covariance & Coefficient & Robust, SE \\
\hline Time & $2.928^{* * *}$ & 0.235 \\
\hline Time $^{2}$ & $-1.099^{* * *}$ & 0.101 \\
\hline \multicolumn{3}{|l|}{ Parental rules (ref: 0-2 units of alcohol) } \\
\hline Allowed to drink 3-4 units of alcohol & $1.266^{* * *}$ & 0.145 \\
\hline Allowed to drink 5 units of alcohol or more & $1.722^{* * *}$ & 0.211 \\
\hline No rules regarding alcohol consumption (at parties) & $0.909^{* * *}$ & 0.155 \\
\hline Do not know & 0.382 & 0.352 \\
\hline \multicolumn{3}{|l|}{ Crosslevel interaction } \\
\hline Allowed to drink 3-4 units of alcohol*time & $-0.877^{* *}$ & 0.281 \\
\hline Allowed to drink 5 units of alcohol or more* time & $-1.968^{* * *}$ & 0.391 \\
\hline No rules regarding alcohol consumption* time & $-0.939 * *$ & 0.292 \\
\hline Do not know* time & -0.853 & 0.707 \\
\hline Allowed to drink 3-4 units of alcohol* time ${ }^{2}$ & 0.211 & 0.130 \\
\hline Allowed to drink five units of alcohol or more* time ${ }^{2}$ & $0.641^{* * *}$ & 0.183 \\
\hline No rules regarding alcohol consumption $*$ time $^{2}$ & $0.290^{*}$ & 0.134 \\
\hline Do not know $*$ time $^{2}$ & 0.318 & 0.333 \\
\hline \multicolumn{3}{|l|}{ Gender (ref: female) } \\
\hline Male & $0.472^{* * *}$ & 0.072 \\
\hline Parents' alcohol consumption during weekend (units) & $0.0196^{*}$ & 0.0084 \\
\hline Average household disposable income, natural log & $0.234^{*}$ & 0.096 \\
\hline \multicolumn{3}{|l|}{ Parents agree that teenagers with a proper upbringing } \\
\hline are able to set limits for themselves & $-0.195^{*}$ & 0.086 \\
\hline \multicolumn{3}{|l|}{ Thresholds $(\tau)$} \\
\hline$\tau 1$ & $3.985^{* *}$ & 1.165 \\
\hline$\tau 2$ & $4.832^{* * *}$ & 1.170 \\
\hline$\tau 3$ & $5.489^{* * *}$ & 1.174 \\
\hline$\tau 4$ & $6.075^{* * *}$ & 1.177 \\
\hline \multicolumn{3}{|l|}{ Random effects } \\
\hline Random intercept & $1.301^{* * *}$ & 0.229 \\
\hline Random slope & $0.198^{*}$ & 0.079 \\
\hline Covariance parameter & $-0.416^{* * *}$ & 0.112 \\
\hline Number & 2,584 & \\
\hline
\end{tabular}

${ }^{*} p<0.05,{ }^{* *} p<0.01$, and ${ }^{* * *} p<0.001$. Only variables that are significant at the $5 \%$ level are included in the model.

between the random slopes and intercepts is significant and negative (-0.42), we can conclude that during a period of 10 years, young people who drink heavily at baseline lower their binge drinking frequency more compared to those who drink less heavily at baseline. In other words, young people may start out with quite different binge drinking patterns but across time, they become more similar.

We also find that the cross-level interaction between parents' rules and time is statistically significant, meaning that parents' rules are associated with young people's binge drinking trajectory from age 15 to $25 / 26$. But because young people with lenient rules already binge drink frequently at this age, we find that across time they expe- rience a less steep rise in binge drinking compared to young people whose parents had strict rules. Thus, in accordance with our second hypothesis, young people who at age 15 were allowed to drink either 3-4 units or 5+ units increased their frequency in binge drinking less over time compared to young people who were allowed to drink a maximum of 2 units of alcohol.

To test whether young people with different parental rules can be statistically differentiated from each other at age $15,18 / 19$, and $25 / 26$, we calculated the marginal effects (holding all other variables at the means) on the probability of binge drinking once, twice, three, or four times during the previous month. Figure 1 shows the marginal effects at age $15,18 / 19$ and $25 / 26$ on the prob- 


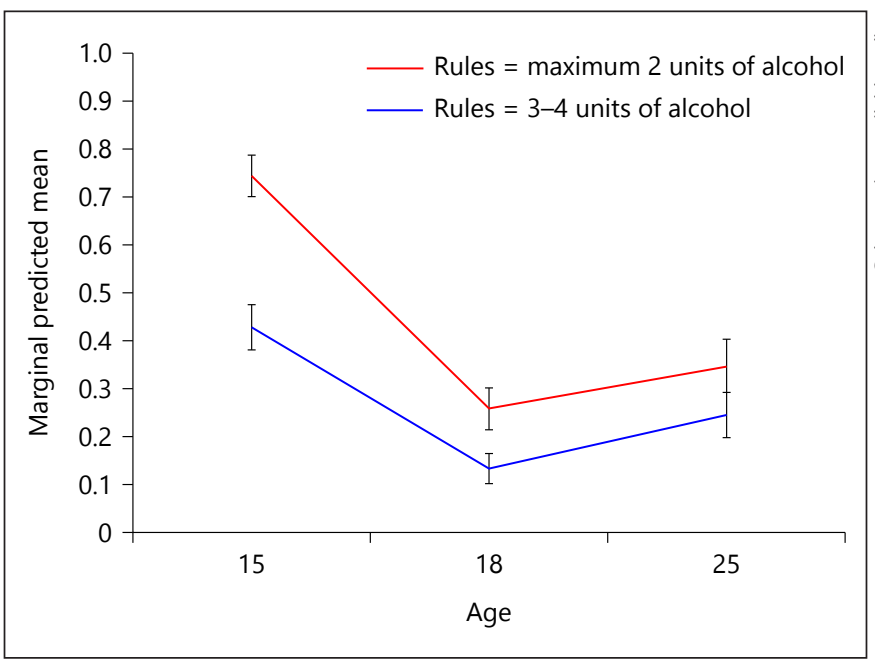

Fig. 1. The probability of binge drinking 0 times for young people whose parents have strict versus lenient rules. The marginal effects are calculated holding all other variables at their means.

ability of binge drinking 0 times during the previous month among young people who were allowed to drink either a maximum of 2 units of alcohol or 3-4 units at age $15{ }^{1}$ Figure 2 shows the same but for a different binge drinking outcome, namely, the probability of binge drinking 4 times or more during the previous month.

The 2 figures reveal a systematic difference in young people's frequency of binge drinking depending on their parents' rules, but only at age 15 and age 18/19 can we statistically differentiate the 2 groups from each other. Thus, $74 \%$ of young people whose parents had strict rules did not binge drink during the previous month before the interview at age 15 , whereas $43 \%$ of young people with more lenient rules (3-4 + units) abstained from binge drinking (Fig. 1). At age 18/19, 25\% of the young people with strict rules abstained from binge drinking as compared to $13 \%$ among young people with more lenient rules and the difference is still statistically significant. However at age $25 / 26$, we can no longer statistically differentiate the frequency of binge drinking among groups of young people with different alcohol-specific rules.

We see the same pattern in Figure 2. Only $2 \%$ of the young people with strict rules (max 2 units) reported binge drinking 4 times during the last month at age 15,

${ }^{1}$ If we collapsed the alcohol-specific rule "allowed to drink $5+$ " units with "allowed to drink 3-4 units" the result was the same. However, due to few observations in the category "allowed to drink 5+ unites" (at baseline less than $10 \%$ of the parents agreed to this and thus the number of observations decreases a lot across time) we chose to report the result for 3-4 units.

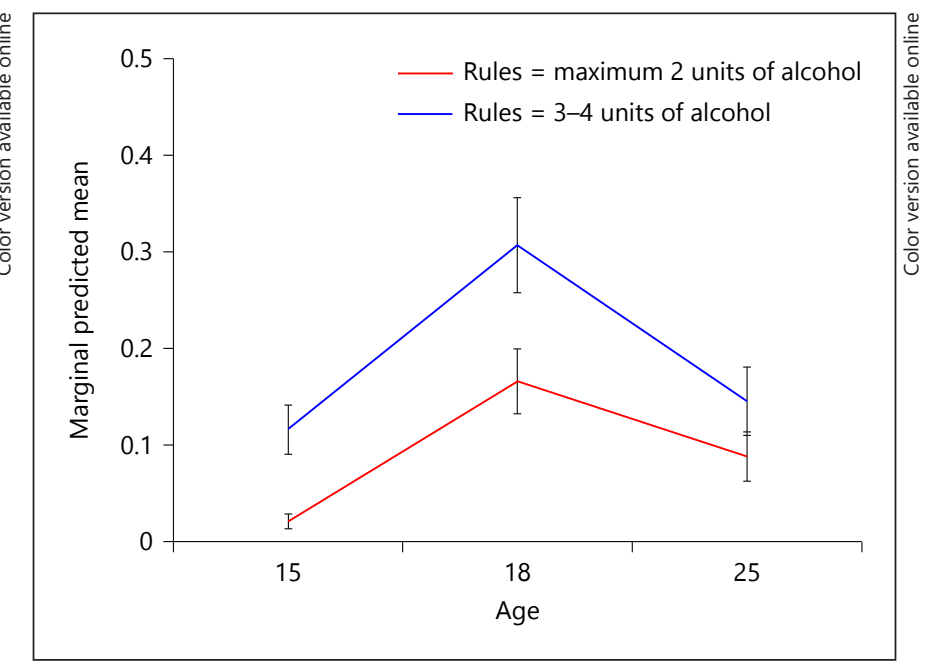

Fig. 2. The probability of binge drinking $4+$ times for young people whose parents have strict versus lenient rules. The marginal effects are calculated holding all other variables at their means.

compared to $12 \%$ among 15 -year-olds with more lenient rules. At age 18/19, the proportion of frequent binge drinkers was $16 \%$ in the group with strict rules and $31 \%$ in the group with more lenient rules. At age 25/26, however, the drinking patterns among young people converge. There is still a tendency for the 25/26-year-olds with restrictive rules to binge drink less, but when we calculate the marginal effects (holding all other variables at the means), the difference is not statistically significant for all outcomes of binge drinking.

Finally, we tested whether categorising parents' "strict" rules differently (e.g., separating the rule 0 units from the other categories) would have changed our results. In general, this was not the case. However, when we calculated the marginal effect, holding all other variables at their mean, we did find that young people whose parents said they were not allowed to drink at all were more likely than all other youths to abstain from binge drinking at age 15 (analysis not shown but can be sent on request).

\section{Discussion}

This paper analysed the development of young people's binge drinking (5+ units of alcohol on one occasion) with the help of longitudinal survey data $(2005,2008$, 2015). The general pattern was that heavy episodic drinking increased from the first (age 15) to the second (age 18/19) measure point after which it decreased somewhat again (age 25/26). Especially, frequent binge drinking 
(3 times a month or more) was more common among 18/19-year-olds (40\%) than among 25/26-year-olds (24\%). The development from 2008 to 2015 corresponds to the phenomenon "maturing out", described in international research where young people's drinking has been shown to peak in their early 20s, after which it declined when they take on new responsibilities, in terms of employment and family relations [30, 31, 34, 35]. Contrary to findings in previous research, maturing out among our respondents was not significantly related to their occupational status (employment, unemployment, studies), nor was it associated with background characteristics such as parents' education or divorce.

Our analyses showed that "strict" alcohol rules at age 15 were associated with lower rates of heavy episodic drinking across time also when we controlled for socioeconomic background, parents' drinking and attitudes to upbringing. Hence, over a time span of 10 years, young people whose parents allowed them to drink a maximum of 2 units of alcohol at age 15 consistently had a more moderate drinking practice than youths whose parents allowed them to drink 3 units of alcohol or more.

Furthermore, when analysing the marginal effects of rule-setting we found that the difference in frequent binge drinking between young people with lenient versus strict rules is statistically significant at age 15 and age $18 / 19$. At age 15, regular binge drinking (4+ times a month) was almost non-existent among youths allowed to drink a maximum of 2 units while it was practiced by $12 \%$ among youths allowed to drink 3-4 units. At age 18/19, twice as many young people were regular binge drinkers in the group with lenient rules as compared to the group with strict rules.

However, at age 18/19, we also found that the effect of parents' alcohol rules began to diminish (for similar results in a Dutch context, see [9]). At this point in their life, most young Danes drink alcohol with the purpose of getting intoxicated [36]. There are strong norms among adolescents in Denmark favouring drunkenness - and tying popularity to intoxication-focused drinking $[37,38]$. It is therefore not surprising that cautious drinkers tend to adapt their alcohol consumption to their more heavy drinking peers, and consequently, that the differences between the groups with different rules diminish. Furthermore, around age $18 / 19$, many young people start moving away from home and are no longer under the daily influence of their parents, including the influence of alcohol rules and alcohol monitoring. At age 25/26, when many young people have "matured out" of their heavy adolescent drinking, we can no longer statistically differentiate

A Longitudinal Study of Parents' Influence on Young People's Drinking Trajectories young people's binge drinking frequency in relation to their parents' alcohol-specific rules when we calculate the marginal effects.

Finally, we want to mention the main strengths and weaknesses of our study. The highlight of this research is that it was based on a longer follow-up period (10 years) than most other studies addressing the relationship between parental rules and adolescent drinking. As mentioned in the introduction, the majority of studies in this field are cross-sectional or longitudinal, covering 2-3 years only. Another strength of our study is that we conducted surveys with both adolescents and parents in 2005, which means that the information about alcohol rules and attitudes on upbringing, as well as on parents' alcohol use, was derived from another source (the survey with parents in 2005) than the information about adolescent drinking (which came from the surveys with young people in 2005, 2008 and 2015). Furthermore, because information about education and income is often insufficient in surveys, we supplemented our survey data with data from administrative registers where information about socioeconomic factors is detailed and precise.

One limitation of the study is attrition. The combined response rates in the initial youth survey (2005) and the parental survey (2005) was relatively high $(n=1,292$ (65\%) but conditioned on parents' alcohol-specific rules, and with time the response rate diminished to 873 young people in 2008 and 784 in 2015 . However, we did not find that respondents with high frequencies of binge drinking in 2005 or respondents coming from families with relatively lenient alcohol rules were less likely to participate in the surveys in 2008 and 2015.

Another limitation of this study is that we only asked parents about their rules and attitudes to upbringing at baseline. Although previous research has shown that age 15 is a significant moment for how young Danes "learn to drink alcohol" [36], it is likely that parents' alcohol rules change when their children get older - and it is also probable that variations in when and how this change happens have an influence on the further development of young people's drinking. Thus, future studies could benefit from investigating how changing parental rules influence children's drinking trajectories, and how young people's increased drinking over time influence their parents' alcohol rules [39]. Likewise, our study did not investigate how lenient alcohol rules might be associated with the development of decidedly problematic drinking habits, that is, addiction [40] and/or binge drinking with severe physical, emotional or social consequences. 
When considering our findings, one may ask whether it is the rules themselves that have an effect on young people's drinking or if the association between rules and drinking is a reflection of something else, for instance the family's socialisation patterns in general, personal characteristics of the children or closeness vs. distance of the parent-child relationship. Previous studies have addressed several of these factors, such as adolescents' personality type, their attachment to parents, their relationships with peers and so on, showing that parental alcohol rules have an effect even when these factors are controlled for $[8,12,13]$. Our study included questions about parents' attitudes to upbringing meant to capture the family's socialization ideals in general, not only their attitudes to alcohol (see methods section above). However, only one of these questions was significantly related to the young people's drinking in 2005-2015, namely, the statement "adolescents who have received a good upbringing are able to set their own limits for their behavior". This indicates that parents who are successful in limiting their adolescents' binge drinking do not only set alcohol-specific rules for their children; they are also characterized by a general belief in upbringing (in relation to drinking as well as in other life spheres), not the least in order to help adolescents develop self-control (for a further discussion on this, see [21]).

Two conclusions for prevention policy may be drawn from our study. First, parents should be informed that alcohol-specific rule-setting has an effect, at least in the age span 15 to $18 / 19$. Denmark is the European country with the smallest proportion of the population being nondrinkers [15]. We therefore regard it as unlikely that larger groups of Danish parents would like to ban adolescent drinking altogether. There is a strong cultural association between drinking and partying, general socialization and "having fun" in Denmark that most parents do not want their children to refrain from. What we have shown in the present paper, though, is that a parental rule of maximum 2 units of alcohol (as compared to 3 units or more) curbs adolescent binge drinking, not the least continuous binge drinking every weekend which for some young people may be associated with long-term harm. Second, our study indicates that parental rule-setting alone cannot prevent heavy occasional drinking in the long run. For this aim, broader efforts are needed - for instance school campaigns, agreements between groups of parents, alcohol rules at youth parties and a change in the alcohol legislation. After all, Denmark is one of the few European countries where 16-year-old youth can drink alcohol legally and our analysis shows that there is a great pressure towards drinking to intoxication particularly during the age period 15 to $18 / 19$. Hence, more initiatives are needed to support the position of adolescents who do not drink with a focus on drunkenness and to make their peers accept and respect their status as non-drinkers/ non-bingers.

\section{Conclusion}

This paper showed that parental alcohol rules for 15-year-olds have a preventive effect on the development of young people's drinking patterns. Strict alcohol rules are related to less binge drinking - and especially less frequent binge drinking - also when we control for factors such as socioeconomic background, parental drinking and parental attitudes to upbringing. With time, however, adolescents from families with strict alcohol rules close in on their peers' alcohol use. The conclusion is, therefore, that parental rules matter, also in a drunkenness-focused alcohol culture like the Danish.

\section{Acknowledgement}

Furthermore, we would like to acknowledge that this work was supported by grants from the Rockwool Foundation for the project Young Adults, Drugs and Alcohol (YODA) - a 10-year longitudinal study with Professor Margaretha Järvinen as the Principle Investigator. We would also like to thank Peter Rohde Skov (VIVE) for suggesting the statistical model and the referees for valuable comments.

\section{Disclosure Statement}

The authors have no conflicts of interest to disclose. The research also complies with legal and ethical guidelines for human studies in Denmark. Such a statement is mentioned in the manuscript.

\section{References}

1 Reifman A, Barnes GM, Dintcheff BA, et al: Parental and peer influences on the onset of heavier drinking among adolescents. J Stud Alcohol 1998;59:311-317.

2 Turrisi R, Jaccard J, Taki R, et al: Examination of the short-term efficacy of a parent intervention to reduce college student drinking tendencies. Psychol Addict Behav 2001;15: 366-372.

3 Abar C, Abar B, Turrisi R: The impact of parental modeling and permissibility on alcohol use and experienced negative drinking consequences in college. Addict Behav 2009;34: 542-547. 
4 Stafström M: Influence of parental alcoholrelated attitudes, behavior and parenting styles on alcohol use in late and very late adolescence. Eur Addict Res 2014;20:233-240.

5 Latendresse SJ, Rose RJ, Viken RJ, et al: Parenting mechanisms in links between parents' and adolescents' alcohol use behaviors. Alcohol Clin Exp Res 2008;32:322-330.

6 Guo J, Hawkins JD, Hill KG, Abbott RD: Childhood and adolescent predictors of alcohol abuse and dependence in young adulthood. J Stud Alcohol 2001;62:754-762.

7 Van Der Vorst H, Engels RC, Dekovic M, et al: Alcohol-specific rules, personality and adolescents' alcohol use: a longitudinal personenvironment study. Addiction 2007;102: 1064-1075.

8 Janssen T, Larsen H, Peeters M, et al: Interactions between parental alcohol-specific rules and risk personalities in the prediction of adolescent alcohol use. Alcohol Alcohol 2014; 49:579-585.

9 Van Der Vorst H, Burk WJ, Engels RC: The role of parental alcohol-specific communication in early adolescents' alcohol use. Drug Alcohol Depend 2007;111:183-190.

10 Van Den Eijnden R, Mheen Dv, Vet R, et al: Alcohol-specific parenting and adolescents' alcohol-related problems: the interacting role of alcohol availability at home and parental rules. J Stud Alcohol Drugs 2011;72: 408-417.

11 Mares SH, Lichtwark-Aschoff A, Burk WJ, et al: Parental alcohol-specific rules and alcohol use from early adolescence to young adulthood. J Child Psychol Psychiatry 2012;53: 798-805.

12 McKay MT: Parental rules, parent and peer attachment, and adolescent drinking behaviors. Subst Use Misuse 2015;50:184-188.

13 Miller P, Plant M: Parental guidance about drinking: Relationship with teenage psychoactive substance use. J Adolesc 2010;33:5568.

14 Swedish Council for Information on Alcohol and Other Drugs: The 2003 Espad-report: Alcohol and other drug use among students in 35 European countries. Stockholm, 2004.
15 European Commission: EU Citizens' Attitudes Towards Alcohol. Brussels, 2010, p 24.

16 Demant J, Østergaard J: Partying as everyday life: investigations of teenagers' ieisure life. J Youth Stud 2007;10:517-537.

17 Hawkins JD, Catalano RF, Miller JY: Risk and protective factors for alcohol and other drug problems in adolescence and early adulthood: implications for substance abuse prevention. Psychol Bull 1992;112:64-105.

18 Ryan SM, Jorm AF, Lubman DI: Parenting factors associated with reduced adolescent alcohol use: a systematic review of longitudinal studies. Aust N Z J Psychiatry 2010;44:774783.

19 Stone AL, Becker LG, Huber AM, Catalano RF: Review of risk and protective factors of substance use and problem use in emerging adulthood. Addict Behav 2012;37:747-775.

20 Spijkerman R, van den Eijnden RJ, Huiberts A: Socioeconomic differences in alcohol-specific parenting practices and adolescents' drinking patterns. Eur Addict Res 2008;14:26-37.

21 Järvinen M, Østergaard J: Governing adolescent drinking. Youth Soc 2009;40:377-402.

22 Gundelach P, Järvinen M, (ed): Unge, fester og alkohol. København: Akademisk Forlag, 2006.

23 Järvinen M, Demant J, Østergaard J (ed): Stoffer og natteliv. København: Hans Reitzels Forlag, 2010.

24 Økonomi- og Indenrigsministeriet: Familiernes økonomi. Fordeling, fattigdom og incitamenter. København: Økonomi- og Indenrigsministeriet, 2014, p 191.

25 Muthén BO, Muthén LK: The development of heavy drinking and alcohol-related problems from ages 18 to 37 in a U.S. national sample. J Stud Alcohol 2000;61:290-300.

26 Caswell S, Pledger M, Pratap S: Trajectories of drinking from 18 to 26 years: identification and prediction. Addiction 2002;97:14271437.

27 Caswell S, Pledger M, Hooper R: Socioeconomic status and drinking patterns in young adults. Addiction 2003;98:601-610.

28 Wellman RJ, Contreras GA, Dugas EN, et al: Determinants of sustained binge drinking in young adults. Alcohol Clin Exp Res 2014;38: 1409-1415.

29 Schulenberg J, O’Malley PM, Bachman JG, et al: Getting drunk and growing up: trajectories of frequent binge drinking during the transition to young adulthood. J Stud Alcohol 1996; 57:289-303.

30 O'Malley PM: Maturing out of problematic alcohol use. Alcohol Res Health 2004;28:202204

31 Staff J, Greene KM, Maggs JL, Schoon I: Family transitions and changes in drinking from adolescence through mid-life. Addiction 2014;109:227-236.

32 Rabe-Hesketh S, Skrondal A: Multilevel and Longitudinal Modeling Using Stata (ed 3). Texas, Stata Press, 2012, p 575f

33 Singer J, Willet J: Applied Longitudinal Data Analysis: Modeling Change and Event Occurrence. New York, Oxford University Press, 2003, p 215.

34 Labouvie E: Maturing out of substance use: selection and self-correction. J Drug Issues 1996;26:457-476.

35 Lee MR, Chassin L, Villalta IK: Maturing out of alcohol involvement: transitions in latent drinking statuses from late adolescence to adulthood. Dev Psychopathol 2013;25:11371153.

36 Østergaard J: Learning to become an alcohol user. Adolescents taking risks and parents living with uncertainty. Addict Res Theory 2009; 17:30-43.

37 Järvinen $M$, Gundelach P: Teenage drinking, symbolic capital and distinction. J Youth Stud 2007;10:5-71.

38 Demant J, Järvinen M: Social capital as norms and resources: focus groups discussing alcohol. Addict Res Theory 2011;19:91-101.

39 Otten $\mathrm{R}$, van der Zwaluw CS, van der Vorst $\mathrm{H}$, Engels RC: Partner effects and bidirectional parent-child effects in family alcohol use. Eur Addict Res 2008;14:106-112.

40 Engels RC, Vermulst AA, Dubas JS, Bot SM, Gerris J: Long-term effects of family functioning and child characteristics on problem drinking in young adulthood. Eur Addict Res 2004;11:32-37. 